\title{
The variability of inner ear orientation in saurischian dinosaurs: Testing the use of semicircular canals as a reference system for comparative anatomy
}

The vestibular system of the inner ear houses three semicircular canals—oriented on three nearly-orthogonal planes - that respond to angular acceleration stimuli. In recent years, the orientation of the lateral semicircular canal (LSC) has been regularly used to determine skull orientations for comparative purposes in studies of non-avian dinosaurs. Such orientations have been inferred based on fixing the LSC to a common set of coordinates (parallel to the Earth's horizon), given that the orientation to gravity of this sensory system is assumed constant among taxa. Under this assumption, the LSC is used as a baseline (a reference system) both to estimate how the animals held their heads and to describe craniofacial variation among dinosaurs. However, the available data in living birds (extant saurischian dinosaurs) suggests that the orientation of the LSC in non-avian saurischian dinosaurs could have been very variable and taxon-specific. If such were the case, using the LSC as a comparative reference system would cause inappropriate visual perceptions of craniofacial organization, leading to significant descriptive inconsistencies among taxa. Here, we used Procrustes methods (Geometric Morphometrics), a suite of analytical tools that compares morphology on the basis of shared landmark homology, to show that the variability of LSC relative to skull landmarks is large (ca. $50^{\circ}$ ) and likely unpredictable, thus making it an inconsistent reference system for comparing and describing the skulls of saurischian (sauropodomorph and theropod) dinosaurs. In light of our results, the lateral semicircular canal is an inconsistent baseline for comparative studies of craniofacial morphology in dinosaurs. 
1 Jesús Marugán-Lobón ${ }^{1,2}$ and Luis M. Chiappe ${ }^{2,{ }^{*}}$ and Andrew A. Farke ${ }^{3}$

21 Unidad de Paleontología, Dpto. Biología, Universidad Autónoma de Madrid, 28049

3 Cantoblanco (Madrid). Spain. 2 The Dinosaur Institute, Natural History Museum of Los

4 Angeles County, Los Angeles, CA 90007. U.S.A. 3 Raymond M. Alf Museum of

5 Paleontology, Claremont, California USA

6 * Corresponding Author: Luis M. Chiappe. The Dinosaur Institute, Natural History

7 Museum of Los Angeles County, Los Angeles, CA 90007. U.S.A E-mail:

8 chiappe@nhm.org

9

10

11

12

13

14

15

16

17

18

19

20

21

22

23

24

25

26

27

28

29 Introduction

PeerJ reviewing PDF | (v2013:05:489:1:0:NEW 15 Jul 2013) 
Anatomical reference systems determining the spatial context by which different

31 parts of an animal's body should be compared (horizontal and vertical axes) are

32 essential for comparative anatomical and phylogenetic studies. In anthropology and paleoanthropology, multiple reference systems have been devised to that end, such as Broca's plane (the plane of the optic nerves) and the Frankfurt plane (the orbito-meatal plane) (Gould, 1981). Anatomical studies in primates also use Reid's baseline, a standard reference system in anthropometry that is particularly common in conventional radiography and computer tomography (CT) (Strait \& Ross 1999). Historically, all these morphological reference systems have been established relative to a stereotyped head posture (when at rest or in alert) and Earth's gravity, yet not surprisingly, none of them is entirely congruent to the others. When orthodontists use one reference framework, be it Reid's plane or the Frankfurt plane, the system is fortunately relatively stable because anatomical variation at an intra-specific (human) scale is small. However, important inconsistencies arise when extrapolating the use of these anatomical reference systems at larger taxonomic scales.

Lebedkin (1924) was the first to suggest that the lateral semicircular canal (LSC) within the inner ear could serve as a proxy to estimate at rest or alert head postures, and de Beer (1947) argued that angular deviations between the LSC and the Earth's horizon in such a stereotyped posture are small enough to support such assumption. This notwithstanding, de Beer also found important inconsistencies for this rule, since the method could not be applied to humans, in which the deviation of the orientation of the LSC and the Earth's horizon at such stereotype postures is nearly $37^{\circ}$ (Fig. 1).

52 Subsequently, striking differences in the position of the labyrinths in the skulls of different species of birds led van der Klaauw (1948) to point out the possibility of a functional relationship between the position of the labyrinth and a stereotyped head posture. To test this hypothesis, Duijm (1951) undertook an inter-specific study through direct observations on living birds, and like de Beer previously, demonstrated that the craniofacial anatomy was best compared when skulls were oriented according to the animals' alert posture (Marugán-Lobón and Buscalioni, 2006). Nonetheless, this study also documented inconsistencies between the orientation of the LSC and that of the skull in such a stereotyped posture - the LSC showed a broad rotational spectrum when the skulls were oriented in the alert posture (Figs $1 \mathrm{~b}$ and 2). This notwithstanding, the author endorsed the use of a horizontal placement of the LSC as an anatomical proxy for the way in which birds hold their heads in such postures. Just as de Beer had reasoned previously, Duijm advocated that the angular deviation of the LSC from a horizontal position (approximately $-19^{\circ}$ to $30^{\circ}$ relative to the horizon while the skull is in the alert position) was smaller than that of any other skull structure and that the mean orientation was close to $0^{\circ}$ (Fig. 2).

68 De Beer and Duijm's seminal works were inspiring, and have been widely followed not 69 just in the context of interpretations of how extinct animals held their heads but also as 70 a reference system for descriptive morphology and anatomical comparisons (e.g., 71 Rogers 1998, Sampson \& Witmer 2007, Sereno et al. 2007, Evans 2006, Witmer \& 72 Ridgely 2009, Witmer et al. 2008). For instance, using the orientation of the LSC 73 Sereno et al. (2007) estimated the 'alert' head posture of the rebbachisaurid

74 Nigersaurus, discussing that this animal's head was bizarre because its face was PeerJ reviewing PDF | (v2013:05:489:1:0:NEW 15 Jul 2013) 
oriented vertical to the ground. However, the estimation of head posture from the LSC is very imprecise (Duijm 1951) and if this is used as a reference system, it can lead to descriptive inconsistencies, as Taylor, Wedel \& Naish (2009) rightfully argued. Take the human head as an example; using the LSC as a reference system (Fig. 1) one would describe the anatomy of the human skull bent forwards and downwards, as if the person looked on to the ground at a point one or two meters away (Girard 1923), yet clearly, the human face is not anatomically sloped downwards. Implicitly, all these comparisons entail that the LSC is oriented differently relative to the components of the human skull, and that such may likely be the case of the sauropod Nigersaurus. Thus, if the orientation of the LSC is potentially unpredictable and problematic as an anatomical reference system, is there an alternative approach that can allow anatomical comparisons of the skulls to be standardized? Here we argue that methods of mathematical shape analysis are suitable for that purpose, in particular the Procrustes methods of geometric morphometrics.

Procrustes methods are widely applied in comparative morphology (Adams Slice \& Rohlf, 2004) and more recently, they have been used as a tool to improve the comparative study of animal behavior by filtering out uninformative body postures (Fureix et al. 2011). In the field of morphological research, Procrustes methods are part of the field of Geometric Morphometrics (GM), and their effectiveness relies on the comparison of configurations of biologically definable - anatomically homologousCartesian coordinates of points (a.k.a. landmarks) involving mathematical operations rather than concepts rooted in biological intuition or classical morphology, such as the use of recognizable postures and fixed comparative baselines (Zelditch et al., 2012). To this end, GM compares configurations of 2D and 3D biologically homologous landmarks within a common reference coordinate system (the statistically computed mean configuration; Chapman 1990, Rohlf \& Slice 1990; Bookstein 1991, Adams, Slice $\&$ Rohlf 2004). Such a procedure is accomplished by a least-squares estimation of translation, rotation (posture), and scaling parameters that help to optimally superimpose the landmark configurations without altering their original topology.

Here, using GM and a case study in saurischian dinosaurs, we assess whether there is any discrepancy between the orientation of skulls according to the LSC and that based on a system of coordinates provided by craniofacial landmark homology. In light of our findings, we argue that landmark homology provides a more consistent and easier to standardize anatomical reference system for depicting and comparatively studying anatomical systems than do classic reference systems such as the orientation of the LSC. 
116 der Klaauw 1948). The experiment involved three steps: (1) determining a stereotyped

117 head posture in live birds, (2) measuring and describing skull morphologies in the

118 stereotyped posture, and finally (3) testing if such measurements allowed estimation of

119 the stereotyped posture from the orientation of the LSC (i.e., if there is a relationship

120 between LSC orientation and head posture). The first step involved measuring the

121 orientation of the head in several species of birds at the Amsterdam's zoo and in the

122 field. These measurements were performed with binoculars equipped with a graduated

123 arc in the lenses, a plumb-rule (used to test verticality), and a hairline to indicate the

124 horizon. The baseline in the animal's head was the ventral edge of the beak. The

125 second step was to replicate the observed alert head posture of the studied species

$126 \quad(n=32)$ in the lab, and it was achieved by reorienting the beaks of their respective skulls

127 to the corresponding degrees measured in the stereotyped alert posture, relative to the

128 horizon. Then, the skulls were dissected through the midline, and the orientations of

129 the cranial floor, the clivus (i.e., the basioccipital bone), the foramen magnum, and the

130 LSC, were all measured and analyzed. The third step focused on discussing the

131 observed variability in orientation of the LSC in the stereotyped alert posture, which

132 was less random (i.e., apparently more normally distributed) than that of the other

133 variables, yet highly variable between extremes (ca. $50^{\circ}$ ). In the original paper, all of

134 these data are provided as skull sketches of each species with labeled lines denoting

135

136

137 the angular orientation of the skull structures in the specific alert posture (Duijm 1951; Figure 3, p. 208). We recovered the angular data and generated Fig. 1b (the stork's head posture in alert), and Fig. 2 (polar histogram). To this end, we scanned that figure with a high-resolution flatbed scanner (at 300ppp) and measured the schemes using the digital protractor utility of TPSdig2 (2.16; Rohlf 2010).

We studied a sample of saurischian dinosaurs that embraces a broad range of skull shape disparity (Marcus, Hingst-Zaher \& Zaher 2000) and thus, of potential semicircular canal orientations (Duijm 1951). Digital pictures corresponding to 16 taxa of saurischian dinosaurs and one extant crocodile in lateral view were studied in two dimensions (Table 1). CT-scan information on the orientation of the LSC was taken from the literature for only 10 of these skulls (Table 1). Additionally, the orientation of the LSC for the stork, while in alert posture, was taken from Duijm's data. On each specimen in the sample, we digitized the coordinates of 5 homologous landmarks (Fig. 3a; see caption for their anatomical description). The landmark configurations homogeneously cover the entire skull (facial skeleton and cranium) to guarantee that

151 (Marugán-Lobón \& Buscalioni 2004). Landmarks were selected to be visible across a

152 variety of taxa with disparate cranial anatomy. Ideally, the landmarks should be

153 coplanar in 2D to avoid the effect of foreshortening, and our choice of coordinates is

154 significantly close to this requirement, as it is on most studies of skull geometry that

155 use GM. The only exception is landmark 4, but the variation of this coordinate should 156 not alter the results since it is anatomically medial and sagittal, and therefore restricted 
157 to vary only in a single 2D plane, comparable to that of the rest of landmarks (i.e., it will

158 not change coronally).

The landmark coordinates were aligned by a Generalized Procrustes Analysis

160 (Gower, 1973) using the program Morpheus et al. (Slice 2002). This method is the

161 standard in GM and allows comparison of 2D or 3D configurations of landmarks within

162 a common reference system (Fig. 3b), which is statistically estimated as the mean from

163 the superimposition of the configurations after optimally minimizing the distances between homologous landmarks. This optimal superimposition is performed by translating, scaling and rotating the coordinates without altering the original distances between the landmarks (i.e., the topology of the configuration). The configurations are first brought to a common coordinate system that by consensus is the average configuration (or Grand Mean). The configurations are then rigidly scaled to the same size (i.e., isometric scaling), and they are subsequently rotated over the shared geodesic centroid. The residual mismatch and irreducible distance among homologous landmarks after the Procrustes alignment is due to the geometric differences between the configurations (after translation, rotation and scaling have been filtered out), and is known as Procrustes shape data; such data is suitable for further multivariate analyses. Importantly, these newly obtained data are 'invariant' to (i.e., it does not possess any information about) translation, scale and rotation (i.e., posture) (see also; Slice 2007, Mitteroecker and Guntz, 2009, Viscosi and Cardini 2011, Zelditch et al. 2012).

In most cases, the landmarks were digitized in lateral view in arbitrary orientations. However, in those specimens for which the LSC orientation within the skull was known, the landmark data were digitized with the LSC set at $0^{\circ}$ (i.e., the LSC horizontal, see Table 1). Once the configurations have been aligned with GM any change in the orientation of the latter skulls corresponds to an angular change in the orientation of the LSC and can be measured. To be consistent with Duijm's (1951) data, we maintained the author's notation of positive and negative values above and below the horizon, respectively. Importantly, the Procrustes data is invariant to translation, scale and rotation, and the way to depict the superimposed configurations is arbitrary. A logical orientation thus is often selected to neatly illustrate the results. Here we chose to orient the superimposed data according to the skull of Crocodylus johnstoni, digitized as if resting horizontally with its ventral surface on a flat surface (Fig. 2a) (Witmer et al. 2008). Given that the body plan of Crocodylus johnstoni is characteristically dorsoventrally compressed, there is little doubt about what is dorsal, ventral, anterior or posterior relative to Earth's spatial directions (i.e., the vector of gravity and the horizon).

the Procrustes method, will nearly share the same spatial coordinates relative to the

194 Earth's axes. Furthermore, in such a posture the orientation of the LSC of Crocodylus

195 johnstoni is nearly co-planar with the horizon (Witmer et al. 2008). Therefore, any

196 angular differences in the semicircular canal's orientation that may result from the

197 Procrustes superimposition (differences in the orientation of the LSC of each dinosaur 
taxon with respect to the horizontal one of Crocodylus) can be intuitively visualized. It is important to stress, however, that this way of depicting the results is as arbitrary as any other, it does not alter the results, and importantly, it does not have any functional meaning (i.e., postural).

In order to compare the geometric similarity between skulls we used a phenetic clustering algorithm (the unweighted pair group method, UPGMA (Rohlf \& Sokal 1981)) on the shape data, and compared this result with a UPGMA classification of the landmark configurations recovering rotational-postural-information (i.e., using the orientation of the LSC as a reference system in those specimens where this information was available).

Results

When the skulls are oriented relative to the Procrustes mean, the average orientation of the LSC with respect to the horizon is approximately $-9^{\circ}$ (Std. Dev. $=16.96^{\circ}$; Confidence interval $\pm 95 \%=[11.67 ; 29.31]$; Fig. 3), although this mean value may not be particularly informative given that deviations involve complementary (i.e., positive or negative) orientations (Mardia, 1972). Comparing the extremes (the prototyped skull of the rebbachisaurid Nigersaurus and the theropod Incisivosaurus), the total range of degrees of deviation relative to the horizon is approximately $55.6^{\circ}$ (Range [Maximum $=-52^{\circ}$, Minimum $\left.=4.5\right]$ ), which is nearly equivalent to the range of LSC orientations documented for living birds when their head is in the alert posture (approximately $50^{\circ}$ [2]; compare Figs. 2 and 4). The rebbachisaurid sauropod Nigersaurus is an outlier yielding a notable negative skewness to the distribution of LSC in saurischians. Although some skull postures after the Procrustes superposition do not differ much from the postures in which the LSC is horizontal, others differ more notably, and this is obviously due to the fluctuating orientations of their LSCs relative to their craniofacial geometry (Table 1). For instance, the skulls of Tyrannosaurus and Majungasaurus remain in a nearly identical position (i.e., its LSC remains nearly parallel-less than $1^{\circ}$ - to the ground in both instances). In Allosaurus and Camarasaurus there is also a very slight deviation from their original posture $\left(2.3^{\circ}\right.$ and $-4.0^{\circ}$, respectively), whereas in the owl and in Diplodocus this deviation is definitively higher $\left(-9.3^{\circ}\right.$ and $\left.-12.5^{\circ}\right)$. In other skulls such as those of Nanotyrannus and Nigersaurus (both largely reconstructed fossils) the orientation of the canals changes significantly, $\left(-22.3^{\circ}\right.$ and $-51.0^{\circ}$, respectively). Although there is a tendency in the sampled dinosaurs to pitch up the LSC relative to the horizon, in Incisivosaurus the LSC is pitched down $4.5^{\circ}$ (Table 1 ).

The phenetic clustering algorithm (UPGMA) on the Procrustes shape data finds two well defined groups differentiable on the basis of morphology (i.e., the orientation 
237 (including the prototyped skull of Nigersaurus) group together, and a parallel

238 association happen with the skulls of the two tyrannosaurids in the sample

239 (Tyrannosaurus and Nanotyrannus, the latter possibly a juvenile T. rex), which are also

240 morphologically very similar to each other. However, all these congruent taxonomic

241 groupings made by the UPGMA on the Procrustes shape data are dispersed if skulls

242 are compared by re-setting the orientation of the landmarks so the LSC is at $0^{\circ}$; Fig. 5 ,

243 right column).

Discussion

Orienting skulls for anatomical comparison using the LSC generally requires both the preservation of the canals, as well as CT scan data of sufficient quality to reliably delineate these structures. These criteria are not easily or often met (particularly for fossils), and thus finding skull orientations for comparative purposes using GM is more practical in the broadest range of cases, when general skull morphology can be restored. On the other hand, the obtained results stress that the orientation of the LSC of saurischian dinosaurs varies greatly relative to the rest of the skull (Fig. 4) and that such variability is independent of skull geometry (i.e., as in other tetrapods, including birds, there is no fixed alignment between the orientation of the LSC and skull morphology; David et al. 2010). When combined with information from living birds (Duijm, 1951), our results also show that a broad spectrum of LSC orientation remained relatively constant for at least the last 200 million years of dinosaur evolutionary history. In light of this, it is unlikely that the LSC serves as a consistent baseline to describe or compare craniofacial morphologies among these animals. Moreover, its use may lead to heterogeneous anatomical descriptions (Fig. 4), hence introducing inconsistencies when scoring character-states in cladistic analyses and inferring paleobiological attributes.

Before the establishment in GM of the Procrustes methods based on the Least reference system (i.e., to standardize a set of coordinates for location, orientation, and size for comparative purposes) was to use the Two-point registration method (Bookstein 1991), which establishes a fixed baseline between two landmarks. It was soon realized that truly invariant landmarks are extremely rare in complex forms, which entails that fixing any given two landmark locations to zero variance (i.e., as a baseline) inevitably and randomly transfers their true variation throughout the entire system (Zelditch et al. 2012). This situation even worsens if the selected landmarks are too

273 situation takes place when establishing the LSC as a reference system. When a skull is oriented on the basis of aligning the labyrinth to a fixed set of coordinates (i.e., a

275 horizontal LSC), the orientation of the LSC (with all its variability relative to other skull 276 structures) is transferred to the orientation of the entire skull, resulting in an equivocal 
perception of the skull's geometry (i.e., confounding anatomical spatial directions across taxa). For example, using the LSC as a reference system, Sereno et al. (2007) envisioned the prototyped skull of the rebbachisaurid Nigersaurus as a very unusual animal because, among other morphological particularities, its rostrum pointed almost vertically towards the ground (Figs. 4 and 5). However, when the skull of Nigersaurus is oriented according to its craniofacial landmark homology with GM, its skull geometry is comparable to that of other sauropods (Fig. 5). Objectively, when viewed from the perspective of GM, it is the orientation of the labyrinth of Nigersaurus (not the head posture or its craniofacial geometry) that is unusual among other saurischians. Furthermore, given that the development of the semicircular canals has a strong genetic component (Jeffery \& Spoor 2004), it is plausible to assume that the orientation of the LSC is case-specific (Billet et al. 2012), although this needs to be further tested in extant taxa.

The use of the LSC as a reference system assumes that the orientation of the semicircular canal matches the coordinate system defined by Earth's gravity (Vinchon et al. 2007), presupposing that every species' stereotyped head posture (at rest or in alert) will be congruent with the vertical axis via the orientation of the LSC (de Beer 1947). However, the fact that in every dinosaurian taxon the LSC does not share the same spatial orientation with respect to Procrustes-aligned craniofacial landmarks and to the horizon challenges that assumption. Such an assumption is also inconsistent with physiological evidence related to vestibular control, which indicates that LSC biomechanics sense angular acceleration stimuli and respond to head motion (David et al., 2010; Fitzpatrick, Butler \& Day 2006). In effect, there is a documented tendency in tetrapods to misalign the semicircular canals with the Earth's axes, which physiologically helps all canals to receive a component of angular acceleration during horizontal head rotations and thus, to actively participate in producing horizontal compensatory movements during motion (Cohen \& Raphan 2004). This vestibular control is jointly guided within the cerebellum through information provided by visual pathways specialized to detect translational visual flow (Wylie, Bischof \& Frost 1998; Wylie and Frost 1999; Van de Water 2012). Furthermore, the characteristic physical organization of the labyrinth in three dimensions renders the canals biomechanically insensitive to the direction of gravity (Rabbitt, Damiano \& Grant 2004) and that function is restricted to the otolith within the vestibular system.

In most anatomical descriptions of archosaur skulls, the plane of reference used to align skulls for comparison is not specified. Judging by figures in many of these studies, this is most typically done by orienting the maxillary tooth row horizontally (e.g., Sampson et al., 2010; Horner and Goodwin, 2009; Campione and Evans, 2012). In order to be consistent in anatomical descriptions, here we recommend that, (1) the frame of reference for aligning anatomical axes should be stated, and (2) that the axes obtained using the geometric morphometric methods described here are easily 
318 the possibility of estimating how fossil organisms held their heads (i.e., how the head

319 was carried), an issue that needs to be further studied in living organisms, they clearly

320 show that the horizontal alignment of the LSC cannot provide a consistent anatomical

321 reference system for skull comparisons in dinosaurs, and possibly in other tetrapods.

322 Instead, we argue that by analyzing homologous morphological landmarks, Geometric

323 Morphometrics offers a more consistent anatomical reference system, one that is

324 independent of posture and purely based on homologous anatomical variables.

326 Acknowledgements

327 We thank C. Mehling (American Museum of Natural History, New York) and D. Unwin

328 (Museum für Naturkünde, Berlin) for specimen access and A. Balanoff for the CTs

329 scans of Incisivosaurus. We are also grateful to S. Abramowicz and W. Evans for

330 creating the artwork and editing the manuscript, respectively. J. Lobón-Cerviá, A.

331 Buscalioni, and N. Martínez-Abadías provided helpful comments to the manuscript and

332 L. Witmer and M. D'Emic reviewed an earlier version of the paper-all these

333 discussions strengthened our study. Funding for this research was provided by a

334 Collection Study Grants of the American Museum of Natural History (New York), the

335 MECD/Fulbright Postdoctoral Mobility Program (Spain), project DGCYT

336 CGL2009_11838 BTE from the Ministerio de Economia y Competitividad (Spain), and

337 the Dinosaur Institute of the Natural History Museum of Los Angeles County (Los

338 Angeles).

339

340 References

34Adams DC, Slice DE, Rohlf FJ. 2004. Geometric morphometrics: ten years of progress

342 following the "revolution"._Italian Journal of Zoology 71:5-16.

34Billet G, Hautier L, Asher R, Schwarz C, Crumpton N, Martin T, Ruf I. 2012. High

344 morphological variation of vestibular system accompanies slow and infrequent

345 locomotion in three-toed sloths. Proceedings of the Royal Society B: Biological

346 Sciences 279:3932-3939.

34Bookstein FL. 1991. Morphometric tools for landmark data: geometry and biology. New

348 York: Cambridge University Press.

34Yampione NE, Evans DC. 2011. Cranial growth and variation in edmontosaurs

350 (Dinosauria: Hadrosauridae): Implications for latest Cretaceous megaherbivore

351 diversity in North America. PLoS ONE 6(9):e25186. doi:10.1371/journal.pone.0025186.

35Xarr TD. 1999. Craniofacial ontogeny in Tyrannosauridae (Dinosauria, Coelurosauria).

353 Journal of Vertebrate Paleontology 19(3):497-520.

354hapman, R. E. 1990. Conventional Procrustes approaches. In: Rohlf FJ, Bookstein FL, 355 eds. Proceedings of the Michigan morphometrics workshop, (Special Publication).Ann

356 Arbor: University of Michigan Museum of Zoology, 251-267. 
35Tohen B, Raphan T. 2004. The physiology of the vestibulo-ocular reflex (VOR). In:

358 Highstein SM, Fay RR, Popper AN, eds. The vestibular system. New York: Springer

359 Verlag, 235-285.

36(David R, Droulez J, Allain R, Berthoz A, Janvier P, Bennequin P. 2010. Motion from the 361 past. A new method to infer vestibular capacities of extinct species. Comptes Rendus

362 Palevol 9:397-410.

36łle Beer GR. 1947. How animals hold their heads. Proceedings of the Linnean Society of 364 London 159:125-139.

36\$)lattre D, Fénart R. 1960. L'Hominisation du crane [The hominization of the cranium]. 366 Paris: Editions CNRS.

36Duijm MJ. 1951. On the head posture in birds and its relation features. Proceedings of the 368 Koninklijke Nederlandse Akademie van Wetenschappen. Series C 54:202-271.

36 Evans DC. 2006. Nasal cavity homologies and cranial crest function in Lambeosaurine 370 Dinosaurs. Paleobiology 32:109-125.

37Fénart R. 1996. Répartition spatiale des volumes faciaux. Etude ontogénique et 372 phylogénique en orientation vestibulaire. Bulletin de l'Association des anatomistes 373 (Nancy) 80:13-16.

374itzpatrick RC, Butler JE, Day BL. 2006. Resolving Head Rotation for Human Bipedalism. 375 Current Biology 16(15):1509-1514.

376-fureix C, Hausberger M, Seneque E, Morisset S, Baylac M, Cornette R, Biquand V, 377 Deleporte P. 2011. Geometric morphometrics as a tool for improving the comparative 378 study of behavioural postures. Naturwissenschaften 98(7):583-92.

379

380Girard, L., 1923. Le plan des canaux semi-circulaires horizontaux considéré comme plan 381 horizontal de la tête. Bulletin et Mémoires de la Societe d'Anthropologie de Paris 382 Series 7 (IV), 14-33.

383ould SJ. 1981. The mismeasure of man. New York: W. W. Norton \& Company, p. 448 384Gower JC. 1975. Generalized Procrustes Analysis. Psychometrika 40:33-51.

386ower JC. 2010. Procrustes methods. Wiley Interdisciplinary Reviews: Computational 386 Statistics. 2(4):503-508.

387torner JR, Goodwin MB. 2009. Extreme cranial ontogeny in the Upper Cretaceous 388 dinosaur Pachycephalosaurus. PLoS ONE 4(10):e7626.

389 doi:10.1371/journal.pone.0007626. 
391ebedkin S. 1924. Uber die Lage des Canalis semicircularis bei Säugern. Anatonzischer 391 Anzeiger, 58:449-460.

392leffery N, Spoor F. 2004. Prenatal growth and development of the modern human 393 labyrinth. Journal of Anatomy 204:71-92.

394klaauw van der C. J. 1948. Size and position of the functional components of the skull. A 395 contribution to the knowledge of the architecture of the skull, based on data in the 396 literature. Archives Néerlandaises de Zoologie 9: 1-176.

39 Marcus LF, Hingst-Zaher E, Zaher H. 2000. Application of landmark morphometrics to 398 skulls representing the orders of living mammals. Hystrix 11(1):27-47.

39Marugán-Lobón J, Buscalioni AD. 2004. Geometric morphometrics in macroevolution: 400 morphological diversity of the skull in modern avian forms in contrast to some theropod 401 dinosaurs. In: Eleewa A, ed. Morphometrics in Paleontology and Biology. Heidelberg: 402 Springer Verlag, 157-173.

40Marugán-Lobón J, Buscalioni AD. 2006. Avian skull morphological evolution: exploring exo404 and endocranial covariation with two-block partial least squares. Zoology 405 109(3):217-230.

40\$itteroecker, P. and Guntz, P. 2009. Advances in morphometrics. Evolutionary Biology 407 36:235-247.

40\&abbitt RD, Damiano ER, Grant JW. 2004. Biomechanics of the semicircular canals and 409 otolith organs. In: Highstein SM, Fay RR, Popper AN, eds. The vestibular system. New 410 York: Springer Verlag, 153-202.

41Rogers SW. 1998. Exploring dinosaur neuropaleobiology: viewpoint computed tomography 412 scanning and analysis of an Allosaurus fragilis endocast. Neuron 21:673-679.

41Rohlf FJ, Slice DE. 1990. Extensions of the Procrustes method for superimposition of 414 landmarks. Systematic Zoology 39:40-59.

41Rohlf FJ, Sokal RR. 1981. Comparing numerical taxonomic studies. Systematic Zoology 416 30:459-490.

41 Sampson SD, Loewen MA, Farke AA, Roberts EM, Forster CA, Smith JA, Titus AL. 2010. 418 New horned dinosaurs from Utah provide evidence for intracontinental dinosaur 419 endemism. PLoS ONE 5(9):e12292. doi:10.1371/journal.pone.0012292.

42(Sampson SD, Witmer LM. 2007 Craniofacial anatomy of Majungasaurus crenatissimus 421 (Theropoda: Abelisauridae) from the Late Cretaceous of Madagascar. Journal of 422 Vertebrate Paleontology 27(sp8):32-102. 
42 Sereno PC, Wilson JA, Witmer LM, Whitlock JA, Maga A, Ide O, Rowe TA. 2007. Structural 424 extremes in a Cretaceous dinosaur. PLOS ONE 2(11): e1230.

425 doi:10.1371/journal.pone.0001230.

42(slice DE. 2002. Morpheus et al. Department of Ecology and Evolution, SUNY-Stony Brook, 427 U.S.A; http://morphometrics.org/morpheus_et_al/.

42\$lice DS. 2007. Geometric morphometrics. Annual Review of Anthropology 36:261-81.

42\$trait DS, Ross CF. 1999. Kinematic data on primate head and neck posture: Implications 430 for the evolution of basicranial flexion and an evaluation of registration planes used in 431 Paleoanthropology. American Journal of Physical Anthropology 108:205-222.

43Jaylor MP, Wedel MJ, Naish D. 2009. Head and neck posture in sauropod dinosaurs 433 inferred from extant animals. Acta Palaeontologica Polonica 54(2):213-220.

434/inchon M, Pellerin P, Pertuzon B, Fénart R, Dhellemmes P. 2007. Vestibular orientation 435 for craniofacial surgery: application to the management of unicoronal synostosis. 436 Child's Nervous System 23:1403-1409.

43Xiscosi V, Cardini, A. 2011. Leaf Morphology, Taxonomy and Geometric Morphometrics: A 438 Simplified Protocol for Beginners. PlosOne 6(10) e25630.

439 doi:10.1371/journal.pone.0025630.

440Nater van der TR. 2012. Historical aspects of inner ear anatomy and biology that underlie 441 the desing of hearing and balance prosthetic devices. The Anatomical Record 442 195:1741-1759.

443Neishampel DB, Dodson P, Osmólska H. 2004. The Dinosauria. 2nd edition. Berkeley: 444 University of California Press, 833.

44SNitmer LM, Chatterjee S, Franzosa J, Rowe T. 2003. Neuroanatomy of flying reptiles and 446 implications for flight, posture and behavior. Nature 425: 950-953.

44XNitmer LM, Ridgely RC. 2009. New Insights into the brain, braincase, and ear region of 448 Tyrannosaurs (Dinosauria, Theropoda), with implications for sensory organization and 449 behavior. The Anatomical Record 292:1266-1296.

450Nitmer LM, Ridgely RC, Dufeau DL, Semones MC. 2008. Using CT to peer into the past: $4513 \mathrm{D}$ visualization of the brain and ear regions of birds, crocodiles, and nonavian 452 dinosaurs. In: Endo H, Frey R, eds. Anatomical Imaging: Towards a New Morphology. 453 Tokyo: Springer-Verlag, 67-88.

454Nylie DRW, Bischof WF, Frost BJ. 1998. Common reference frame for neural coding of 455 translational and rotational optic flow. Nature 392:278-282. 
456Wylie DRW, Frost BJ. 1999. Complex Spike Activity of Purkinje Cells in the ventral uvula 457 and nodulus of pigeons in response to translational optic flow. Journal of 458 neurophysiology 81(1):256-266.

45qelditch ML, Swiderski DL, Sheets HD. 2012. Geometric morphometrics for biologists: A 460 primer. Second Edition. Academic Press, p. 478.

461

462

463

464

465

466

467

468

469

470

471

472

473

474

475

476

477

478

479

480

481

482 Figure Legends 
483 Figure 1. Differences in reference systems in skulls. (A) In the human skull there is a

$48430^{\circ}$ difference between the Frankfurt plane and that of the LSC, thus yielding

485 substantially different head orientations (from de Beer, 1947). (B) When a stork is in

486 alert its LSC is oriented $19^{\circ}$ above the horizon, thus when putting the LSC at $0^{\circ}$

487 (horizontal) head posture differs from its alert posture (from Duijm, 1951).

489 Figure 2. Schematic depiction of the semicircular canals and polar histogram of LSC 490 orientations in extant birds, measured in alert posture. The semicircular canals are 491 three interconnected tubes that define three nearly orthogonal planes, and are part of 492 the bony labyrinth of the inner ear. The measurements were obtained relative to the 493 Earth's horizon by Duijm (1951) when the birds were in a stereotyped alert posture at a 494 zoological garden $(n=29)$. Notice that the orientation of the lagena and the cochlear 495 duct in the vestibular apparatus normally varies among species; in our scheme they are steady for simplicity. Although the average orientation was close to zero, LSC angles when birds are in alert approximately ranges from $-19^{\circ}$ to $30^{\circ}$ relative to the horizon.

Figure 3. Landmarks and Procrustes alignment of dinosaur skulls. (A) Example of the configuration of $p=5$ landmarks in lateral view of the skull of a crocodile (C. johnstoni), as it leans on its mandibles over a flat surface. Landmarks are: 1-tip of premaxilla, 2-margin of nasal opening closer to tip of premaxilla, 3-margin of nasal opening further from tip of premaxilla, 4-junction between supraoccipital and parietal at cranial roof, 5-mandibular articulation of quadrate. In this crocodile the orientation of the LSC relative to the horizon is $\sim 0^{\circ}$ (Witmer et al., 2008). (B) Superimposed configurations of landmarks using Geometric Morphometrics (Generalized Procrustes methods, or GPA). The enlarged black landmarks correspond to the Procrustes mean (the consensus reference system). The $x-y$ crosses at each landmark are depicted to illustrate the concomitant correspondence with the spatial directions determined by the morphological plan of the crocodile relative to the Earth's axes. However, note that these skull orientations do not necessarily correspond to head postures in the live specimens.

514 Figure 4. Distribution of estimated measurements after Generalized Procrustes

515 Analysis (GPA) of semicircular canal orientations for the studied dinosaur skulls. In the 516 distribution, the maximal range of angular variance spans between the skull of

517 Nigersaurus $\left(\sim-52^{\circ}\right)$ and that of Incisivosaurus $\left(\sim 4.5^{\circ}\right)$. The schematic skulls are shown

518 in the posture obtained by the Procrustes alignment, and LSC orientations are

519 measured relative to the horizon and as the difference between $0^{\circ}$ and the new GPA 520 orientation. Obtained LSC orientations for all dinosaurs after GPA are listed in Table 1. 
522 Figure 5. UPGMA phenograms grouping dinosaur skulls by geometric similarity.

523 Separate columns illustrate the different skull postures obtained using Procrustes

524 methods (left column) or by aligning the LSC with the horizon (right column). The

525 branching diagram on the right column groups skulls comparing landmark data for

526 which only translation and scale were filtered out, but not rotation (i.e., this data

527 includes skull posture as morphological information, determined by the LSC set to $0^{\circ}$ ).

528 The large white dots at the nodes are highlighting cases of notable grouping

529 differences, such as considering the skull geometry of Nigersaurus as that of either a

530 sauropod (left) or a bizarre dinosaur (right), and that of Nanotyrannus (presumably a

531 juvenile T. rex; Carr 1999) as different from that of Tyrannosaurus. The grouping in the

532 right column indicates that rotation is a main source of morphological difference among

533 skull geometries. The numbered terminal branches denote the taxa listed in Table 1;

534 the LSC orientation is known for those with an asterisk. 


\section{Figure 1}

Figure 1

Differences in reference systems in skulls. (A) In the human skull there is a $30^{\circ}$ difference between the Frankfurt plane and that of the LSC, thus yielding substantially different head orientations (from de Beer, 1947). (B) When a stork is in alert its LSC is oriented $19^{\circ}$ above the horizon, thus when putting the LSC at $0^{\circ}$ (horizontal) head posture differs from its alert posture (from Duijm, 1951).

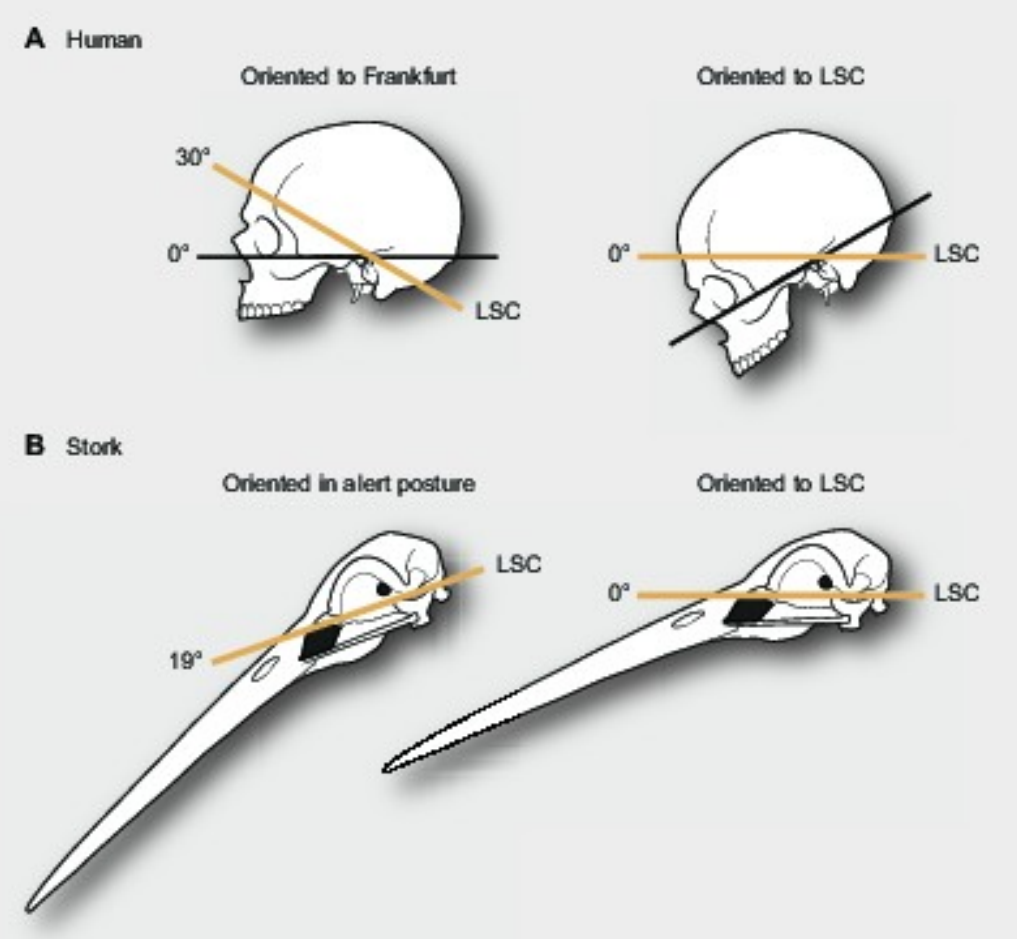




\section{Figure 2}

Figure 2

Schematic depiction of the semicircular canals and polar histogram of LSC orientations in extant birds, measured in alert posture. The semicircular canals are three interconnected tubes that define three nearly-orthogonal planes, and are part of the bony labyrinth of the inner ear. The measurements were obtained relative to the Earth's horizon by Duijm (1951) when the birds were in a stereotyped alert posture at a zoological garden $(n=29)$. Notice that the orientation of the lagena and the cochlear duct in the vestibular apparatus normally varies among species; in our scheme they are steady for simplicity. Although the average orientation was close to zero, LSC angles when birds are in alert approximately ranges from $-19^{\circ}$ to $30^{\circ}$ relative to the horizon.

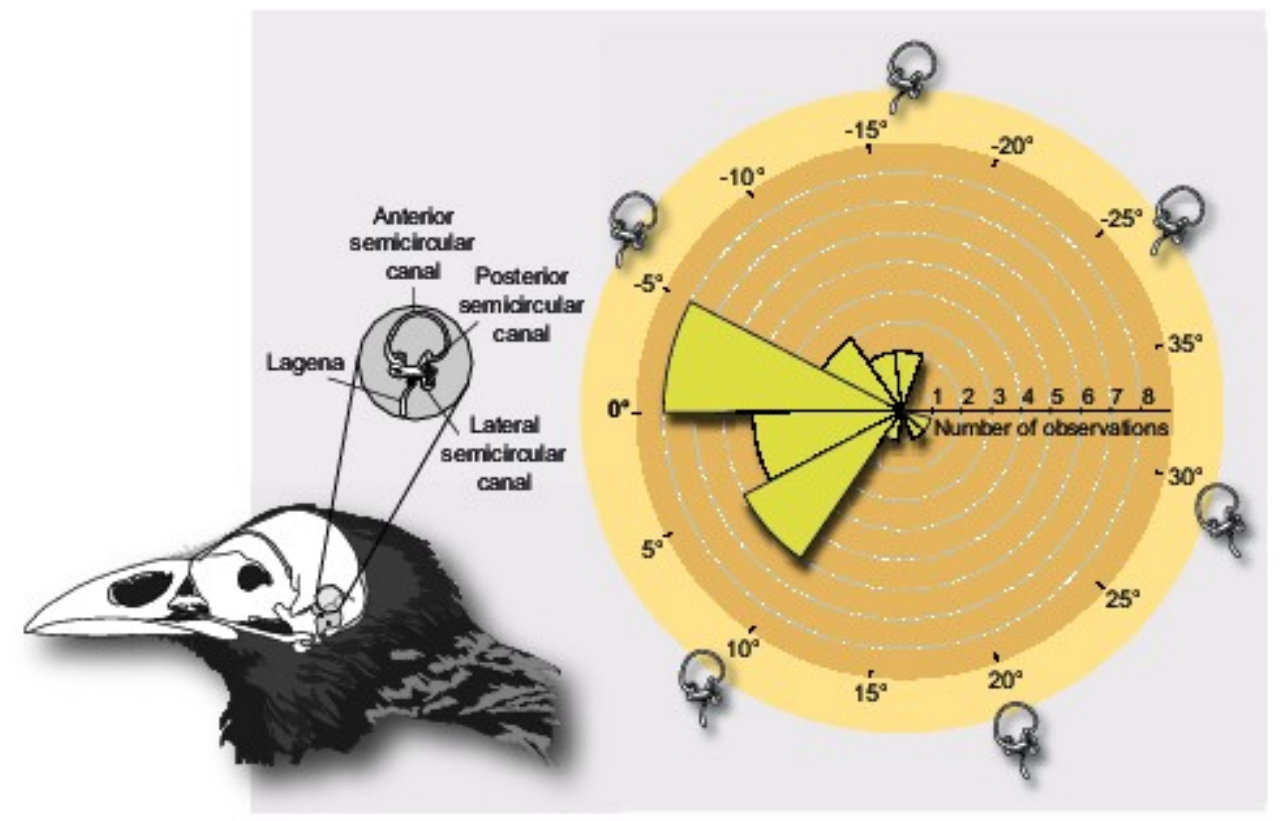




\section{Figure 3}

Figure 3

Landmarks and Procrustes alignment of dinosaur skulls. (A) Example of the configuration of $p=5$ landmarks in lateral view of the skull of a crocodile (C. johnstoni), as it leans on its mandibles over a flat surface. Landmarks are: 1-tip of premaxilla, 2-margin of nasal opening closer to tip of premaxilla, 3-margin of nasal opening further from tip of premaxilla, 4-junction between supraoccipital and parietal at cranial roof, 5-mandibular articulation of quadrate. In this crocodile the orientation of the LSC relative to the horizon is $\sim 0^{\circ}$ (Witmer et al., 2008). (B) Superimposed configurations of landmarks using Geometric Morphometrics (Generalized Procrustes methods, or GPA). The enlarged black landmarks correspond to the Procrustes mean (the consensus reference system). The $x-y$ crosses at each landmark are depicted to illustrate the concomitant correspondence with the spatial directions determined by the morphological plan of the crocodile relative to the Earths's axes.

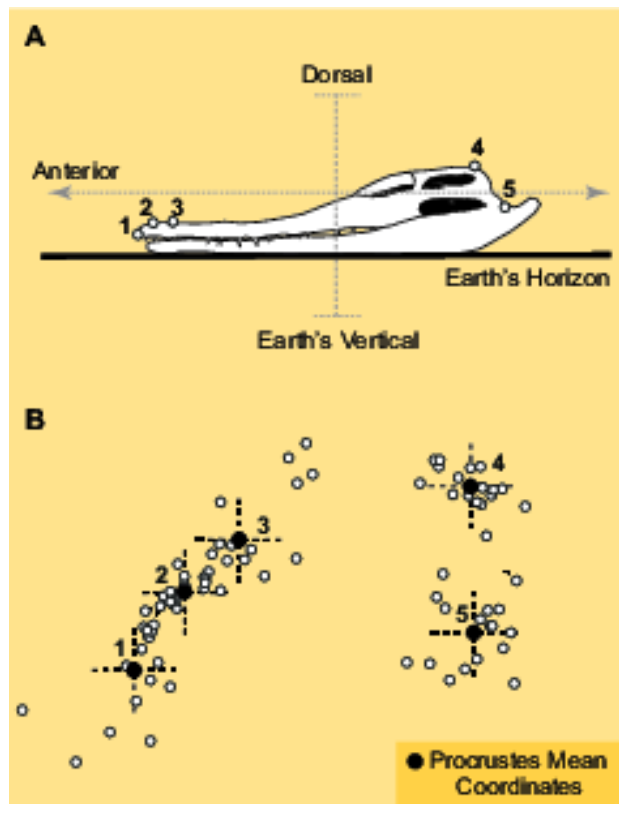




\section{Figure 4}

Figure 4

Distribution of estimated measurements after Generalized Procrustes Analysis (GPA) of semicircular canal orientations for the studied dinosaur skulls. In the distribution, the maximal range of angular variance spans between the skull of Nigersaurus $\left(\sim-52^{\circ}\right)$ and that of Incisivosaurus $\left(\sim 4.5^{\circ}\right)$. The schematic skulls are shown in the posture obtained by the Procrustes alignment, and LSC orientations are measured relative to the horizon and as the difference between $0^{\circ}$ and the new GPA orientation. Obtained LSC orientations for all dinosaurs after GPA are listed in Table 1.

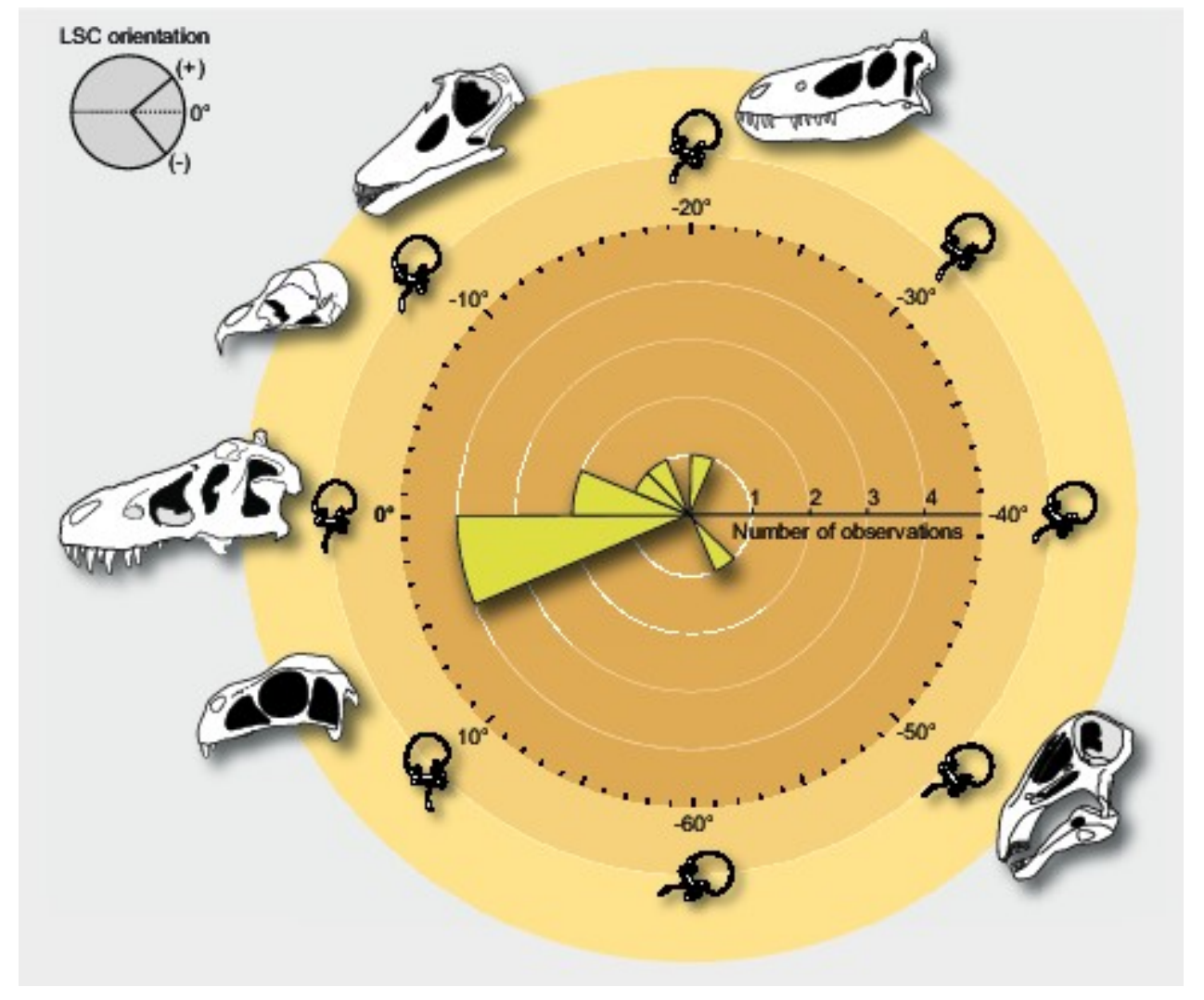




\section{Figure 5}

Figure 5

UPGMA phenograms grouping dinosaur skulls by geometric similarity. Separate columns illustrate the different skull postures obtained using Procrustes methods (left column) or by aligning the LSC with the horizon (right column). The branching diagram on the right column groups skulls comparing landmark data for which only translation and scale were filtered out, but not rotation (i.e., this data includes skull posture as morphological information, determined by the LSC set to $0^{\circ}$ ). The large white dots at the nodes are highlighting cases of notable grouping differences, such as considering the skull geometry of Nigersaurus as that of either a sauropod (left) or a bizarre dinosaur (right), and that of Nanotyrannus (presumably a juvenile T. rex) as different from that of Tyrannosaurus. The grouping in the right column indicates that rotation is a main source of morphological difference among skull geometries. The numbered terminal branches denote the taxa listed in Table 1; the LSC orientation is known for those with an asterisk. 


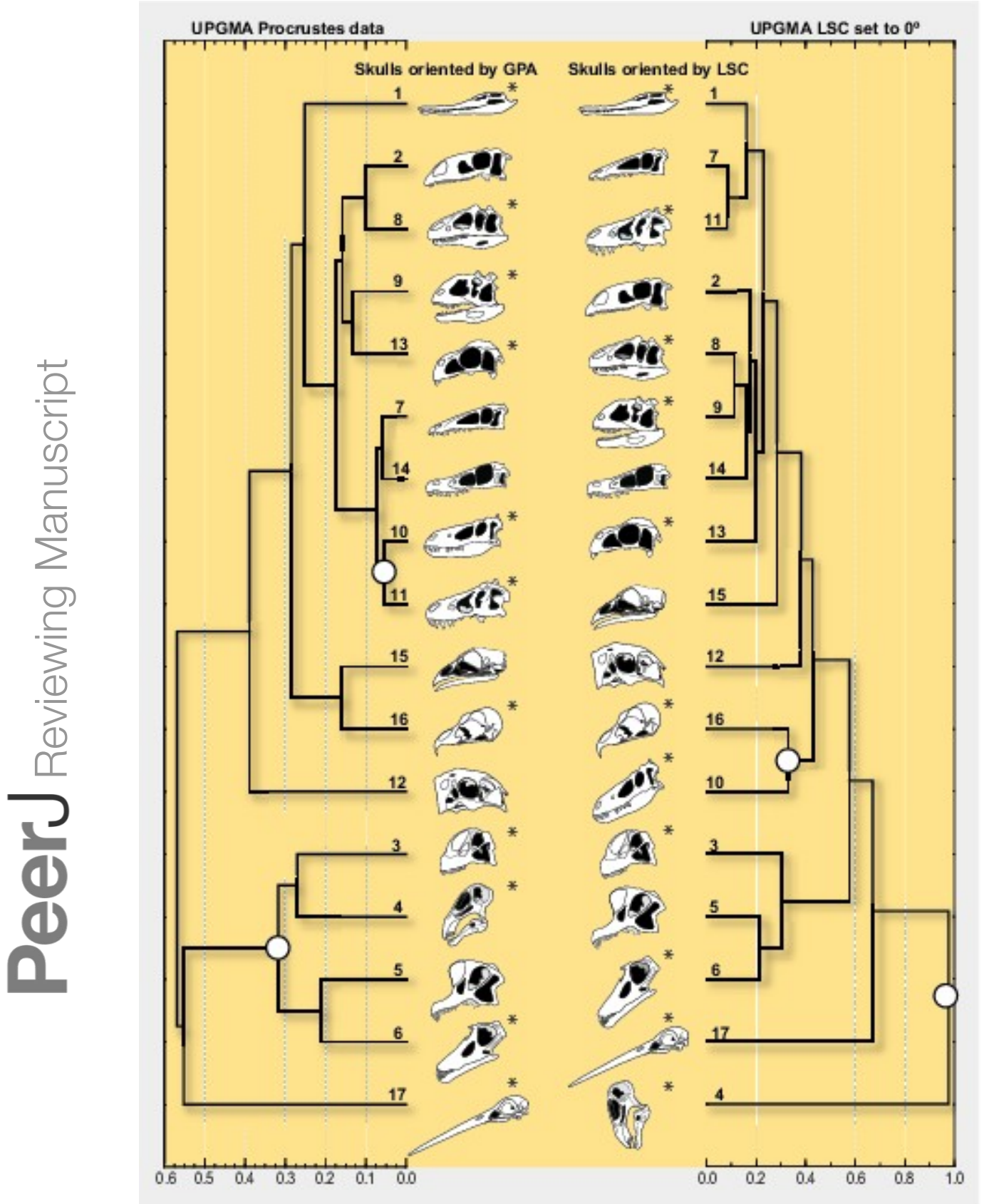




\section{Table $\mathbf{1}_{\text {(on next page) }}$}

Table 1

Studied specimens, collection numbers, and LSC orientations derived from the Generalized Procrustes Analysis. 
Table 1. Studied specimens, collection numbers, and LSC orientations derived from the Generalized Procrustes Analysis.

\begin{tabular}{|c|c|c|}
\hline Specimen & Collection \# & LSC \\
\hline 1 Crocodylus johnstoni & OUVC $10425^{[15]}$ & $0^{\circ}$ \\
\hline 2 Plateosaurus longiceps & MB.R.1937 & - \\
\hline 3 Camarasaurus lentus & CM $11338^{[15]}$ & $-4^{\circ}$ \\
\hline 4 Nigersaurus taqueti & MNN GAD512 ${ }^{[12]}$ & $-51.1^{\circ}$ \\
\hline 5 Giraffatitan brancaii & HMB t1 (S-II) & - \\
\hline 6 Diplodocus longus & CM $11161^{[15]}$ & $-12.5^{\circ}$ \\
\hline 7 Coelophysis bauri & AMNH 480 & - \\
\hline 8 Allosaurus fragilis & UMNH VP $18050^{[14]}$ & $2.3^{\circ}$ \\
\hline 9 Majungasaurus crenatissimus & FMNH PR2100 ${ }^{[11]}$ & $0.2^{\circ}$ \\
\hline 10 Nannotyranus lancensis & CMNH $7541^{[14]}$ & -22.3 \\
\hline 11 Tyrannosaurus rex & AMNH $5117^{[14]}$ & $-1.1^{\mathrm{o}}$ \\
\hline 12 Citpati osmolskae & IGM 100/978 & - \\
\hline 13 Incisivosaurus gauthieri & IVPP V 13326 & 4.6 \\
\hline 14 Velociraptor mongoliensis & AMNH FR6516 & - \\
\hline 15 Gallus sp. & ZMB 77 & - \\
\hline 16 Bubo virginianus & OUVC $10220^{[15]}$ & $-9.3^{\circ}$ \\
\hline 17 Ciconia ciconia & ZMB $253^{[2]}$ & $3.0^{\circ}$ \\
\hline
\end{tabular}

Institutional abbreviations: AMNH; American Museum of Natural History; HMB, MBR and ZMB, Humboldt Museum für Naturkunde; CM=Carnegie Museum; IVPP=Institute of Vertebrate Paleontology and Paleoanthropology; MNN, Musée National du Niger; FMNH, Field Museum of Natural History; OUVC, Ohio University Vertebrate Collections; IGM, Mongolian Institute of Geology; $\mathrm{CMNH}$, Cleveland Museum of Natural History. 